\title{
Improved Bandwidth Utilization based Check pointing Algorithm in Distributed Database
}

\author{
Neera Batra and Manpreet Singh \\ M. M. Engineering College, M. M. University \\ Mullana, Ambala \\ Haryana, India
}

\begin{abstract}
In this paper, we present an optimal-bandwidth, min-process coordinated check pointing algorithm suitable for network failure prone applications in distributed systems. In the developed algorithm, during normal computation message transmission, dependency information among clusters is recorded in the corresponding cluster head processes. When a check pointing procedure begins, the initiator from a cluster concurrently sends composite message to all the cluster head processes which after extracting individual messages from it, further multicasts individual messages to the corresponding currently active receiving processes in their corresponding clusters thus resulting in reduced transmission delay and communication cost, better bandwidth utilization and faster speed of execution. Quantitative analysis shows that proposed algorithm works efficiently in terms of better response time and maximum bandwidth utilization for applications running under critical conditions such as low bandwidth availability and thereby resulting in frequent disconnections.
\end{abstract}

\section{Keywords}

Optimal bandwidth cluster fed check point, non-blocking.

\section{INTRODUCTION}

Cluster federations contain a large number of nodes and are heterogeneous. Nodes in a cluster are often linked by a SAN (System Area Network) while clusters are linked by LANs (Local Area Network) or WANs (World Area Network) [5]. Clusters communicate with each other by message passing. To survive failures, clusters take checkpoints periodically or nonperiodically. Checkpoint is defined as a designated place in a program at which normal processing is interrupted specifically to preserve the status information necessary to allow resumption of processing at a later time. Check pointing is the process of saving the status information. A checkpoint is a snapshot of the state of a process saved on the stable storage which can be reloaded into memory to reduce the amount of lost work in recovery. [6] Checkpoints correspond just to processes states and have no information about the states of the communication channels. Therefore, in order to record a consistent global state of a process, whenever the state of any process indicates that it has sent a message to another process, the state of the receptor must indicate that it has received this message. This requirement is the major condition to the implementation of techniques to record global states of distributed system.

A global checkpoint [8][9][10][14] consists of certain number of checkpoints such that each of these checkpoints corresponds to one of the clusters uniquely in a cluster federation.

In this paper, we specifically address a reduced bandwidth usage and low cost scheme for distributed database based on processes check pointing. Processes take checkpoints periodically managed by the local cluster head and log their output/input in a common table maintained by cluster head. The developed scheme reduces the cluster-to-cluster communication to a single composite message and the cluster head of each cluster is responsible for extracting the individual messages from the composite message and multicast them to the corresponding receiving processes.

The remainder of this paper is organized as follows. In section 2 , we state the data structures used. Section 3 describes the working model of the algorithm. Section 4 presents the proposed check pointing algorithm. In section 5, some relevant observations along with comparison to other algorithms are presented. Section 6 comprises of performance analysis. Section 7 concludes the paper.

\section{DESIGN PRINCIPLES}

\subsection{Models and assumptions}

Application model: Distributed database applications using the composite message model is designed. Processes of this kind of applications are divided into clusters (modules). Processes inside the same cluster communicate a lot while communications between processes belonging to different clusters are limited due to global bandwidth usage resulting in more communication cost incurred and frequent transaction restarts due to unavailability of optimal bandwidth required for Inter-cluster communications.

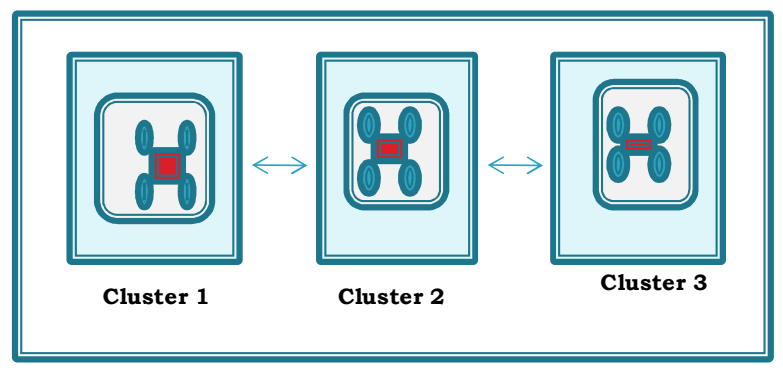

Figure 1: Cluster Federation

\subsection{Architecture model and network assumptions}

We assume a cluster federation as a set of clusters interconnected by a WAN, inter-cluster links being either dedicated or even Internet, or a LAN. Each group of processes may run in a cluster where network links have small latencies and large bandwidths (SAN).

\subsection{Data Structures}

Notations used:
N - Global Bandwidth
$\eta$ - Local Bandwidth
$S N$ - Sequence Number of a Process
$S N^{a}$-Sequence number of cluster $A$
$N_{P}$ - Total number of processes 
$N_{c}$ - Total number of clusters

$\mathrm{CH}$-Cluster Head

$\left\lfloor P_{i, i} Y_{i}\right\rfloor$-Process identity number of $i^{\text {th }}$ process, flag Y

for $i^{\text {th }}$ process

$v_{i}^{a}$ - keeps a record of $S N$ for each process $p_{i}$ in cluster

$A$

$C_{i}(x)^{a}-X^{t h}$ checkpoint of process $i$ in cluster $A$

$Y[i][a]-$ is the flag used to identify active processes at

$x^{\text {th }}$ checkpoint

$t$ - Time taken for a composite message to reach from one $\mathrm{CH}$ to another $\mathrm{CH}$

$p_{1}^{a}(\mathrm{CH})$ - The checkpoint initiating cluster head process cluster $A$

$m^{c}$-control message

$m^{a}$ - Application message

$m^{C a}$ - Composite message

Suppose there are $N_{P}$ processes and $N_{c}$ clusters in the system where $N_{P}$ is much larger than $N_{c}$. Each process is assigned a unique id, $i$ where $\left(1 \leq i \leq N_{P}\right)$.

In our check pointing scheme, for each process in the cluster, the checkpointing dependency information is maintained by its cluster head process. Each Cluster Head $\mathrm{CH}$ sends the composite messages consisting of control and application messages to the cluster head of other clusters which further multicast the message to all the corresponding currently active processes in the cluster after extracting the individual single or multiple messages from the composite message meant for each receiving process. This scheme reduces the message passing and number of lost messages drastically, thus making system more available, reliable and faster and resulting in optimal bandwidth utilization for network failure prone applications.

When a check pointing procedure begins, the sending and the receiving of composite messages is mainly accomplished amongst cluster head processes. To maintain such additional information for each process $\quad p_{i}^{a}$ in cluster $A$, each sending cluster head $\mathrm{CH}$ maintains a $\log$ file $\left\langle p_{i}^{a}, p_{j}^{b}, B\right\rangle$ with $p_{i}^{a}$ as a sending process in cluster $A$ and $p_{j}^{b}$ as a receiving process in cluster $B$ where $\left(1 \leq i, j \leq N_{P}\right)$ and $\left(1 \leq a, b \leq N_{c}\right)$. A vector $v_{i}^{a}$ for keeping a record of $S N$ (Sequence Number) for each process $p_{i}$ in cluster $A$ where flag $Y[i][a]=0$ in case, process $p_{i}$ neither receives or sends any message during current global interval $\left(C_{i}(x)^{a}-C(x-1)^{a}\right)$ at $X^{\text {th }}$ check point. After the global check point is taken, both the fields in the table are set as empty and $S N^{a}$ is incremented.

\section{WORKING MODEL}

In the proposed algorithm, when communication occurs between two processes in different clusters, then dependencies are generated between checkpoints taken in different clusters. Dependencies must be tracked in order to allow the application to be restarted from a consistent state. In our work based on idea adopted from [1], it is the sending process that ensures that none of its sent messages can remain an orphan (received-not-sent).

When the $\mathrm{CH}$ of any cluster initiates the checkpointing procedure by sending the control message to other clusters, then the current cluster's sequence number $S N$ is piggybacked on inter-cluster control message embedded in the composite message which is sent to any active process in any cluster during $X^{\text {th }}$ global checkpoint interval. $\mathrm{CH}$ of each other cluster is responsible for storing these $\mathrm{SN}$ values for synchronization among clusters.

The communication scheme based on message passing from one $\mathrm{CH}$ to other is beneficial only if (i) there are very few chances of message loss due to network failure. So the proposed algorithm works best for the applications which are network failure prone and for applications which do not use secure network media for message communication as it introduces the concept of composite message passing to overcome these two significant shortcomings of communication induced check pointing (ii) $\mathrm{CH}$ communicates the inter-cluster received messages to all the active processes in the cluster within a finite period of time so that there is no synchronization delay. To deal with synchronization delay, the algorithm assumes a threshold value of time interval within which sending $\mathrm{CH}$ creates a composite message comprising of control messages and application messages both and receiving $\mathrm{CH}$ multicasts the received messages to all corresponding processes in the cluster which are participating during current global checkpoint interval $\left(c_{x-1}-c_{x}\right)$, after extracting them from the composite message .

Let us assume that the time taken on an average by a cluster head to send a composite message to other cluster head is a constant $t$ with the assumption that the bandwidth available during message passing remains constant. As seen in most of the previous works[ 1][2][3], If a control message is to be sent to processes in a cluster, time taken by a sending process $p_{i}^{a}$ in cluster $A$ for any processes $p_{j}^{b}$ where $\left(1 \leq p_{j} \leq n\right)$ in cluster $B$ is $t$. If the process $p_{i}$ is supposed to send the control message to each process in cluster $B$ directly, it will take $n^{*} t$ if there are n processes. In the proposed algorithm, the $C H$ of cluster $B$ checks for the value of $Y_{i}$ where $1 \leq i \leq n$ and multicasts extracted control messages and application messages to all the processes with value of $Y_{i}=1$. Suppose time taken by $C H$ to multicast the control message $m^{c}$ and application messages $m^{a}$ among active processes is $\tau$ which is a small fraction of time $t$ as cluster $B$ uses SAN(System Area Network), a very fast and reliable media in comparison to LAN or WAN used for communication amongst clusters. So total time taken by $\mathrm{CH}$ to inform all the active processes for the next checkpoint is $(\tau+t+\tau)$ i.e. $t+2 \tau$ where $\tau$ is time taken by sending process in cluster $A$ to composite the message, $\mathrm{t}$ is the time taken by cluster $A$ to send the message to $C H$ of cluster $B$ and $\tau$ is the time taken by $C H$ of cluster $B$ to extract the individual messages and multicast them to corresponding 
active processes. This value $(t+2 \tau)$ is considered as a threshold value to keep a check on transmission delay caused by $\mathrm{CH}$. Although this threshold value varies during each global check point interval depending upon number of active processes in current global checkpoint interval but this variation is very small, since number of participating processes during each checkpoint interval remain almost constant. Now this threshold value will be a common constant for all the clusters in cluster federation. Hence, each sending and receiving cluster will know a priori about message transmission delay caused by any other $\mathrm{CH}$. So no acknowledgement is required to ensure that cluster head $\mathrm{CH}$ has sent the message to all other processes or not, which belong to same cluster.

Suppose there are two clusters $A$ and $B$ with 4 processes each uniquely identified as $p_{1,} p_{2}, p_{3}, p_{4}$ and $p_{5}, p_{6,} p_{7}, p_{8}$ respectively as shown in Fig. 2 .

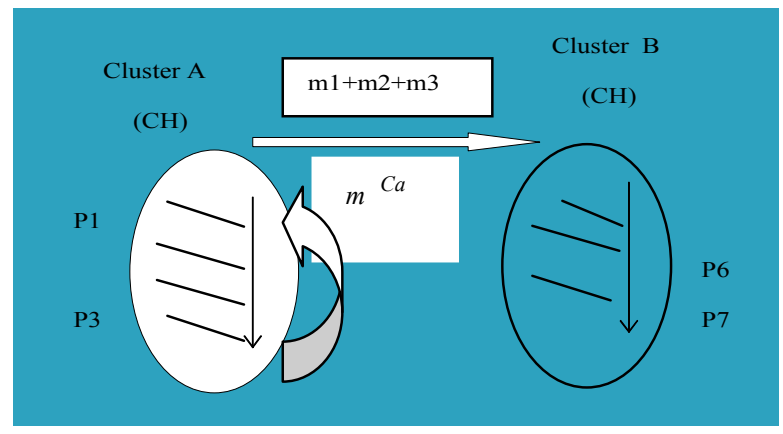

Figure 2. Cluster Communication Through Meassage Passing

Now process $p_{1}$ of cluster $A$ which is initiating cluster head process $p_{1}^{a}(\mathrm{CH})$, when takes a check point, creates a composite message $m^{C a}$ comprising of a single control message with latest $S N$ and application messages received during a fixed time $\tau$ say $2 \mathrm{~ms}$ and then sends this composite message to $C H$ of cluster $B$ in time interval $t$ say 2 ms(micro seconds). $C H$ of cluster $B$ on receiving this composite message $m^{\mathrm{Ca}}$ extracts each individual control message $m^{c}$ and application message $m^{a}$ from it and further multicasts them o all the corresponding receiving processes respectively in cluster $B$ who are active in current global checkpoint interval ' $I$ ' say within $\tau$ ms( say $2 \mathrm{~ms}$ ) .

So total time taken for control message $m^{c}$ sent by cluster $A$ to reach all the active processes in cluster $B=(\tau+t+\tau)=$ $6 \mathrm{~ms}$. Now say after $2 \mathrm{~ms}$ of sending the first composite message by $p_{1}^{a}(\mathrm{CH})$ of cluster $A$, a process $p_{3}^{a}$ belonging to same cluster sends an application message $\left\langle m^{a}, S N^{a}, p_{6}\right\rangle$ piggybacked with $S N^{a}$ along with process identity number of receiving process to cluster $B$ through $p_{1}^{a}(\mathrm{CH})$ embedded in second composite message. $\mathrm{CH}$ of cluster $B$ after extracting the application message $\left\langle m^{a}, S N^{a}, p_{6}\right\rangle$ from the received composite message , sends the message to $p_{6}$ for processing taking total time of $6 \mathrm{~ms}(2+2+2)$ i.e. $(\tau+t++\tau)$ i.e.. $(t+2 \tau)$.Total time taken for processing second composite message = $(2+2+2+2)$ i.e. $(t+3 \tau)=8 \mathrm{~ms}$ where first $2 \mathrm{~ms}$ taken are considered on the basis that this message is sent after $2 \mathrm{~ms}$ of recent global checkpoint interval starts which is $\cong \tau$. Accordingly within $8 \mathrm{~ms}$, all the processes in the cluster come to know about the next global checkpoint to be taken even if they haven't received the first composite message containing the control message in it yet.

On basis of above observations, maximum global checkpoint interval $I=\left(C_{x-1}-C_{x}\right)$ is such that $T=(2+2+2+2)$ i.e. $(t+3 \tau)=8 \mathrm{~ms}$.

The proposed algorithm makes system resilient against any message delay or message loss. Since this threshold value considered is a constant and already known to each cluster, so if any process $p_{1}^{a}(\mathrm{CH})$ of cluster $A$ sends a piggybacked message to cluster $B$, it takes again $t$ time to reach the cluster head $C H$ of cluster $B$ and now the cluster extracts the $S N^{a}$ piggybacked with application message. If $S N^{b}<S N^{a}$, then $C H$ of cluster $B$ informs all the active processes in cluster $B$ about the next checkpoint to be taken and sends the received application message for processing to the concerned process. Therefore instead of waiting for the control message $m^{c}$ to arrive which was embedded in the first composite message, the process $p_{6}$ of cluster $B$ takes a forced checkpoint and updates its $S N$ value with piggybacked $S N^{a}$ value received in second composite message, if $Y[6][b]=1$. The first application message embedded in the second composite message, sent by a $\mathrm{CH}$ to any other cluster, only contains piggybacked information. However, any other process in source cluster doesn't need to piggyback SN value if it sends any other message to the same cluster before the next invocation of the proposed algorithm.

\section{CHECKPOINTING ALGORITHM}

${ }^{*} \mathrm{p}[\mathrm{j}][\mathrm{i}]$ is the $\mathrm{i}^{\text {th }}$ process in $\mathrm{j}^{\text {th }}$ cluster $\&$ we assume $\mathrm{p}[\mathrm{j}][1]$ as cluster head of eack cluster $\mathrm{j}, 1 \leq j \leq N_{c} * \backslash$

$$
N_{p} \geq N_{c} \& N_{p} \in N_{c}
$$

where $\mathrm{N}_{\mathrm{p}}$-Number of processes $\mathrm{N}_{\mathrm{c}}$-Number of clusters

\section{At Sender :}

** Assume $p_{\text {ini }}$ is the initiator in cluster $\mathrm{c}^{*}$

$$
\text { If } p_{\text {ini }}==C H[c]
$$

Step 1: $C H[c]$ takes a checkpoint

Step 2: checks $\mathrm{Y}[\mathrm{k}][\mathrm{c}]==1$ for each process $k$ in Cluster $c$

Step3: $C H[c]$ sends $m^{C a}$ to $C H[j]$ where $C H[j], \forall 1 \leq j \leq N_{c}$.

Step 4: $S N^{c}=S N^{c}+1$; 


\begin{tabular}{|c|c|c|c|c|}
\hline & $\begin{array}{c}\text { Hierarchical } \\
{[3]}\end{array}$ & $\begin{array}{c}\text { Roll- } \\
\text { forward } \\
{[1]}\end{array}$ & $\begin{array}{c}\text { Hybrid } \\
\text { [2] }\end{array}$ & $\begin{array}{l}\text { Proposed } \\
\text { Algorithm }\end{array}$ \\
\hline Cost & $\begin{array}{c}2 * \mathrm{n}_{\text {broad }}+ \\
\mathrm{n}^{*} \mathrm{c}_{\mathrm{air}}\end{array}$ & $\mathrm{n}_{\min } * \mathrm{c}_{\mathrm{air}}$ & $3 n * c_{a i r}$ & $\mathrm{~N}-1 * \mathrm{c}_{\text {air }}$ \\
\hline $\begin{array}{l}\text { Temporary } \\
\text { Checkpoints }\end{array}$ & Yes & No & Yes & No \\
\hline $\begin{array}{c}\text { Non- } \\
\text { blocking }\end{array}$ & Yes & No & Yes & No \\
\hline $\begin{array}{l}\text { No. of } \\
\text { check } \\
\text { points }\end{array}$ & $n_{\text {min }}$ & $n_{\min }$ & $\mathrm{n}+1$ & $n_{\min }$ \\
\hline $\begin{array}{l}\text { Message } \\
\text { complexity }\end{array}$ & $\mathrm{O}(\mathrm{n})$ & $\mathrm{O}(\mathrm{n})$ & $\mathrm{O}(\mathrm{n})$ & $\mathrm{O}(1)$ \\
\hline
\end{tabular}

Step 5: Set $\mathrm{Y}[\mathrm{k}][\mathrm{c}]=0$ for each process $k$ in cluster $c$

\section{At Receiver:}

On receiving $m^{C a}$ from cluster $c$, each $C H[j], \forall 1 \leq j \leq N_{c}$ checks for process $p_{i}$ satisfying condition $Y[i][j]==1, \forall 1 \leq i \leq N_{p}$

Step $1: C H[j]$ after extracting control message $\left\langle m^{c}, S N^{c}\right\rangle$ from $m^{C a}$ sends it to processes with $\mathrm{Y}[\mathrm{i}][\mathrm{j}]==1$.

$$
\begin{array}{ll}
\text { Step 2 : } S N^{j}=S N^{j}+1 ; & 1 \leq j \leq N_{c} \& \\
& C H[c] \notin N_{c}
\end{array}
$$

Step 3: Each extracted $m^{a}$ from $m^{C a}$ is sent to corresponding receiving active process in cluster $\mathrm{j}$ where $\forall 1 \leq j \leq N_{c}$

Step 4: Set $\mathrm{Y}[\mathrm{i}][\mathrm{j}]==0$ for $1 \leq j \leq N_{C}, \quad 1 \leq i \leq N_{p}$ End of algorithm.

\section{IMPLEMENTATION}

It is reasonable to say that the major source of overhead in checkpointing schemes is the response time and network communication latency. Communication overhead becomes a minor source of overhead as the latency of network communication decreases. In this scenario, the coordinated checkpoint becomes worthy since it requires less accesses to stable storage then uncoordinated checkpoints. Moreover, Composite message composition further helps in reducing the network latency significantly thereby resulting in reduced transmission delay, communication cost, better bandwidth utilization and faster speed of executioN.

\section{PERFORMANCE}

The main advantage of our algorithm over the algorithms $[1][2][3]$ is that the inter-cluster transmission delay, communication cost and bandwidth usage remains minimum with increasing number of processes also, thereby making the system more efficient, less prone to network failure and worth using for application running with low strength bandwidth available. We have presented the comparison of performance of the above three algorithms with our algorithm in Table 1. Consider a cluster federation consisting of $n$ clusters where

$\mathrm{N}-\quad$ number of clusters $n_{\min }-\quad$ minimum number of processes that need to take a check point

$C_{\text {air }} \quad$ - $\quad$ cost of sending a message from one process to another

$n_{\text {multi }}$ - time taken to multicast a message to all processes in the system

$n_{\text {broad }}$ - time taken to broadcast a message to all processes in the system

Table 1: Performance Comparison of the Check pointing Algorithms

The cost to complete the checkpointing process using [1] is given as $n_{\text {min }}{ }^{*} c_{\text {air }}$. It is a single phase algorithm and it multicasts only one type of control message. In [2], the initiator sends control messages to all the processes costing $n^{*} c_{\text {air }}$. Then all the receiving processes acknowledge the initiator process about the receiving of control message costing $n^{*} c_{\text {air }}$, then finally the initiator sends all the processes a control message requesting for commit. Hence total cost to complete the checkpointing process using algorithm [2] is given as $\left(3 * n * c_{\text {air }}\right)$.

In [3] the initiator in each cluster broadcasts a check pointing request to all the processes costing $n_{\text {broad }}$. The initiator receives replies from $n$ processes, the cost of which is $n^{*} c_{\text {air }}$. Finally the initiator broadcasts a commit message to all processes to convert their temporary check points to permanent ones, the cost of which is $n_{\text {broad }}$. Hence the total cost of [3] is $n^{*} c_{\text {air }}+2 * n_{\text {broad }}$.

In proposed algorithm, communication takes place directly between clusters. So when one cluster sends a control message to other, it is received by cluster head $\mathrm{CH}$ and multicast to the participating processes in current global checkpoint interval. Since only one control message per cluster is sent and if there are $N$ clusters, one cluster sends $N-1$ messages to all the remaining clusters. Hence the cost is $N-1 * c_{\text {air }}$.

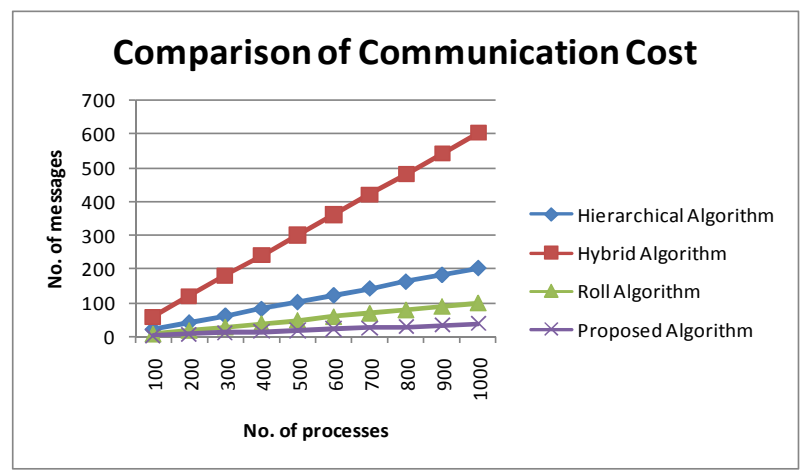

Figure 2. Performance Comparison of Proposed Algorithm with Existing Algorithms

In Fig. 2, the ordinate represents the cost of sending the control messages to complete the check pointing algorithm in the best case for the four algorithms. Fig. 2 clearly demonstrates the better performance of our approach than the 
ones in [1],[2] \& [3] in terms of message passing. In our approach, with the increase in number of processes the number of control messages transferred is minimum. Hence the least chances of traffic congestion and bandwidth utilization

\section{COMPARISON OF EXECUTION TIME}

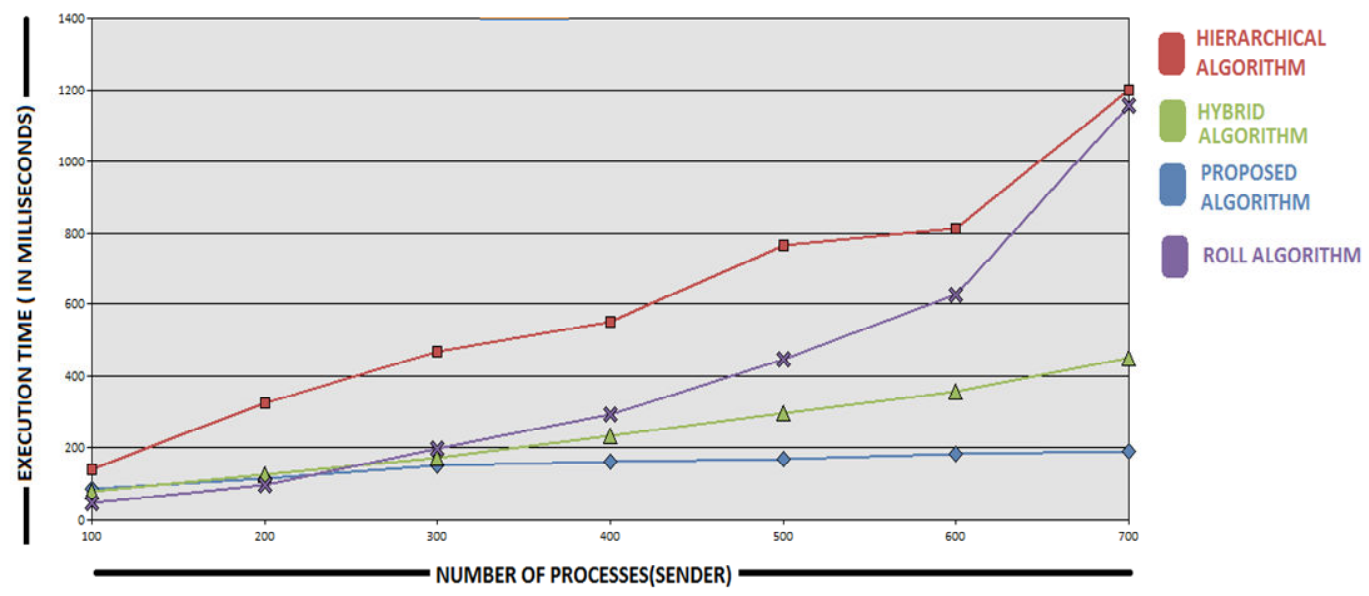

Fig.3 Cpu Utilization Comparison

\section{REFERENCES}

[1] B. Gupta, S. Rahimi, and R. Ahmad, " A New RollForward Checkpointing / Recovery Mechanism for Cluster Federation", International Journal of Computer Science and Network Security, vol. 6, no.11, pp. 292-297, Nov. 2006.

[2] J. Cao, Y. Chen, K. Zhang and Y. He, "Checkpointing in Hybrid Distributed Systems”, IEEE ISPAN'04, 2004.

[3] S. Monnet, C. Morin and R. Badrinath,"A Hierarchical Checkpointing Protocl for Parallel Applcations in Cluster Federations", IEEE IPDPS 2004, 2004.

[4] B. Gupta, S. Rahimi, and Z. Liu, "A New High Performance Checkpointing Approach for Mobile Computing Systems", International Journal of Computer Science and Network Security, vol. 6, no. 5, May 2006.

[5] G. Cao, and M. Singhal, "On coordinated checkpointing in distributed systems", IEEE Transactions on Parallel and Distributed Systems, vol. 9, no. 12, pp. 1213 - 1225, Dec. 1998.

[6] R. Prakash, and M.Singhal, "Low-Cost Check pointing and Failure Recovery in Mobile Computing Systems," IEEE Transactions on Parallel and Distributed Systems, vol. 7, no. 10, pp. 1035-1048, October 1996.

[7] G.Cao, and M. Singhal, "Mutable checkpoints: a new checkpointing approach for mobile computing systems," IEEE Transactions on Parallel and Distributed Systems, vol. 12, no. 2, pp. 157-172, Feb. 2001.
[8] K.M. Chandy, and L. Lamport, "Distributed Snapshots: Determining Global States of Distributed Systems," ACM Transactions on Computer Systems, vol. 3, no. 1, pp. 6375, Feb. 1985.

[9] P. Kumar, L. Kumar, R.K. Chauhan, and V.K. Gupta, "A non-intrusive minimum process synchronous checkpointing protocol for mobile distributed systems," ICPWC 2005, IEEE International Conference on Personal Wireless, vol. 3, no. 1, pp. 63-75, Feb. 1985.

[10] L. M. Silva, and J.G. Silva, "Global checkpointing for Distributed Programs," Proceedings of 11th symposium on Reliable Distributed Systems, pp. 155 -162, Oct. 1992.

[11] B. Gupta, S. Rahimi, and Z. Liu, "A New Non-Blocking Synchronous Checkpointing Scheme for Distributed Systems," Proceeding of $20^{\text {th }}$ International Conference on Computers and Their Applications, pp. 26-31, Mar. 2005.

[12] R. Prakash, and M.Singhal, "Low-Cost Check pointing and Failure Recovery in Mobile Computing Systems," IEEE Transactions on Parallel and Distributed Systems, vol. 7, no. 10, pp. 1035-1048, Oct. 1996.

[13] D. Manivannan, and M. Singhal, "Asynchronous Recovery Without Using Vector Timestamps," Journal of Parallel and Distributed Computing, vol. 62, no. 12, pp. 1695-1728, Dec. 2002.

[14] B. Gupta, S. Rahimi and Yixin Yang, "A Novel Roll-Back Mechanism for Performance Enhancement of Asynchronous Checkpointing and Recovery", Informatica, pp. 1-13, 2007. 